\title{
Mental Health situation in Nepal and priorities for interventions
}

Anupa Rijal ${ }^{*}$

${ }^{1}$ Health Prospect: Journal of Public Health

Received:

4 June 2017

Revised:

7 November 2017

Accepted:

16 January 2018

${ }^{\star}$ Correspondence: anuparijal@gmail.com Health Prospect: Journal of Public Health

\section{Introduction}

We are currently living in a world where one in four people are currently affected by mental disorders, and 3 out of 4 people suffering from mental disorders do not receive any treatments. Neuropsychiatric conditions account $13 \%$ of total disability adjusted life year (DALYs) lost due to all-diseases and injuries and is likely to increase to $15 \%$ with depression accounting for $5.7 \%$ of DALYs by 2030 [1]. The disabilities led by mental disorders like depression, substance abuse, schizophrenia have robbed individuals in realizing their potential, developing coping skills against normal stresses of life, and being economically and socially productive.

The burden is even high for low and middle-income countries like Nepal with less than efficient mental health services- regarding limited diagnostic, treatment and availability of human resources to address mental health issues. Suicide- the second most common cause of death among young people globally; Nepal has seventh highest suicide rate in the world-mostly among girls and woman of reproductive age [2]. Additionally, the aftermath of an earthquake on April 25, 2015, have raised the burden of post-traumatic stress disorders among the survivors exponentially [3-5].

\section{Current Health system provision for addressing mental health in Nepal}

This alarming rate of the mental illness mingled in less than efficient mental health services- availability and accessibility are one of the emerging challenges in Nepal. The stigmatization associated with mental health and pre-labelling of mental health seeker as "mad" has created an enormous barrier in identifying and addressing mental health issues [6]. There are very few specialist mental health services in Nepal ;1 mental hospital and 18 outpatient mental health facilities and 17 generals and teaching hospitals with inpatient mental unit limited to central, zonal or district level hospitals. None of these facilities is specialized for child or adolescents mental health. There is only 0.59 human resource working in mental health per 100,000 populations of which only 0.13 are psychiatrists [7]. At community level counselling and psychotherapeutic are not available and trained mental health force is almost negligible, and those who are trained in mental health are not provided with regular refresher course and are poorly documented. The broad classification of mental health data under "mental disorder" has further limited the evidence of prevalence and management of diverged mental health issues. Moreover, the mental health policy developed in 1997 still lacks a framework for implementation hence fails to integrate mental health within health system in the country [8].

\section{Mental Health Promotion and Prevention in Nepal}

In the era of deinstitutionalization, mental health no longer can be addressed through clinical paradigm alone. This calls for holistic public health approach that not only addresses the multiple determinants of mental health but provides population focused promotion and prevention initiatives. The notion of promotion and prevention with respect to mental health is poorly understood. Mental health promotion deals with enabling individuals, communities and families to strengthen or support positive emotional, cognitive and related experiences. Whereas prevention, as the name suggests, is preventing risk factors and early symptoms of disease improving the overall quality of people. These two approaches are overlapping and are synergistic to each other $[9,10]$.

\section{Addressing mental health through primary prevention in Nepal}

Mental health is a multifaceted problem. So, to address this multi-sectoral approach including non-health sector is critical. The inclusion of mental health in education, disaster preparedness, nutrition programs, maternal and child health seems very timely. Lack of evidence of mental health in Nepalese context is one 
of the significant barriers to identifying and addressing mental health issues in the country. Hence prioritizing mental health in social and clinical research is a must.

Educational institutions and workplace can be a very important catchment area to address mental health issues. Formation child friendly school, inclusion of programs that supports children to speak about stresses, failures and pressures at school level, anti-bullying programs, awareness on drugs and anti-drug programs, provision of counselling sessions for students and training teachers on identifying symptoms and necessary skills to handle such issue at primary level are some of the possible interventions that will create supportive environment against mental health. Moreover, stress prevention program at work settings, provision of physical exercise at work and home settings will help to reduce mental stressors. Studies have shown that physical activity also helps to improve mental health hence there should be provision to include adequate space for school and housing [11,12].

The recent earthquake in Nepal has reflected the need of supportive mental health support setting more than ever. Community-based support group to identify vulnerable group and individual and support in developing healthy coping mechanism is vital in a disaster setting.

Similarly, awareness of mental health issues, would reduce the prevalent stigma around mental illnesses. Developing skills to disclose any problems they have and the problem they are going through in life is equally important. An example of this is Let's Talk initiative of the World Health Organization (WHO) [13].

\section{Reorient health services at primary care level}

Less than $1 \%$ of all health expenditures directed towards mental health $(0.17 \%)$ in Nepal, of which a considerable amount of them are directed towards the only mental hospital, the Patan mental hospital [7]. It is the first specialized mental hospital established in the early 80s. All other hospitals were established after that, and provides either the general or the specialized psychiatric services. The regional and district hospitals provide the both. Theoretically, there is no provision of mental health services at the primary care level or below. Community mental health services are at the mercy of ill-trained health workers who mostly miss recognizing the common symptoms of mental illnesses. The psychosomatic representation such as feeling 'jhum-jhum' is a common neuro-psychiatric complaint among the rural population of Nepal. This kind of neuro-psychiatric illnesses are usually overlooked and most often have been treated for wrong reasons [14]. People with mental illness often seek services for physical illness, failure to identify such psycho-somatic cases will lead to under estimation of the problem. Trained health professional, appropriate referral, investment in mental health to improve the diagnostic procedure is vital.

\section{Psychotropic drugs and its availability}

Unfortunately, there are not any studies on the availability of psychotropic drugs in Nepal. The market dynamics and mark ups in such drugs, according to studies in neighbouring countries shows a high share of branded drugs and high out of pocket expenditure. The annual cost per average case of disorder in Nepal was highest for anti-psychotic drug was 176.47 US\$ [15] and largely such medicines are paid out of pocket. Not having an insurance system complicates the problem furthermore, and there are other hidden costs to the person suffering the illness and their family members due to loss of work days in accessing the services. There are only 12 drugs listed in the national essential medicine list of Nepal related to treatment of mental health problems [16] compared to 17 in 19th revision of WHO essential medicine list (EML) including all the complimentary forms of the medicines as of 2015 [16], and of them, only 3 are present in the free drug list of Nepal [17]. So, our recommendation is that the government without a delay, should allocate essential psychotropic drugs and its formulations to the free drug list.

\section{Conclusions}

Mental health is an emerging health priority though we don't know the exact burden of it as of now. The provision of mental health services at the primary care and tertiary care remains weak with only 0.13 psychiatrists per 100,000 population and 0.59 mental health workers per 100,000 populations. The availability of psychoactive drugs remains faint with high out-of-pocket expenditure on the medicines. The government should immediately roll out mental health services through primary health care, for that number of both specialist and mid-level health workers needs to be trained and their numbers increased. The prevention of mental illnesses should be looked through a primary prevention approach so that the risk factors and their precipitators for poor mental health outcomes at the different levels of community and social structures can be mitigated.

\section{References}

1. Jenkins R, Baingana F, Ahmad R, McDaid D, Atun R. Mental health and the global agenda: core conceptual issues. Mental health in family medicine. 2011 Jun;8(2):69.

2. Nepal's silent epidemic of suicide Cousins, Sophie. The Lancet; London 387.10013(Jan 2, 2016): 16-17.

3. Hagaman AK, Maharjan U, Kohrt BA. Suicide surveillance and health systems in Nepal: a qualitative and social network analysis. International journal of mental health systems. 2016 Jun 6;10(1):46.

4. Whiteford HA, Ferrari AJ, Degenhardt L, Feigin V, Vos T. The global burden of mental, neurological and substance use disorders: an analysis from the Global Burden 
of Disease Study 2010. PloS one. 2015 Feb 6;10(2):e0116820.

5. Drake RE, Binagwaho A, Martell HC, Mulley AG. Mental healthcare in low and middle income countries. BMJ $2014 ; 349$.

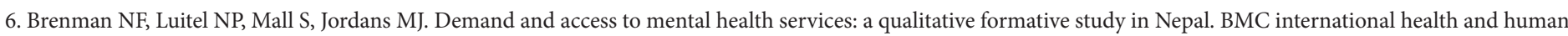
rights. 2014 Aug 2;14(1):22.

7. WHO. WHO-AIMS Report on Mental Health System in Nepal, WHO and Ministry of Health, Kathmandu, Nepal, 2006.

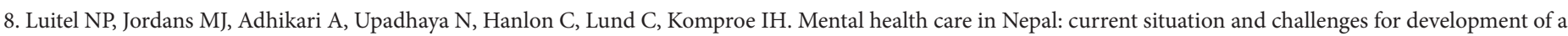
district mental health care plan. Conflict and health. 2015 Feb 6;9(1):3.

9. World Health Organization. Prevention and promotion in mental health. Geneva. 2002

10. World Health Organization. Prevention of mental disorders: Effective interventions and policy options: Summary report. Geneva. 2004

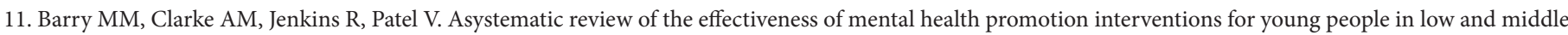
income countries. BMC Public Health. 2013; 13:835.

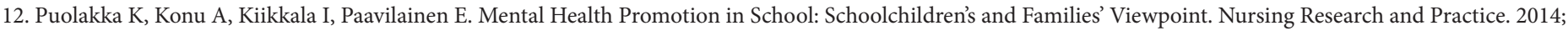
2014:395286.

13. World Health Organization. Depression Let's Talk. 2016. Available from http://apps.who.int/depression-campaign-2017/en/events (accessed 22 May 2017)

14. Kohrt BA. "Somatization" and “Comorbidity”: A Study of Jhum-Jhum and Depression in Rural Nepal. Ethos. 2005 Mar 1;33(1):125-47.

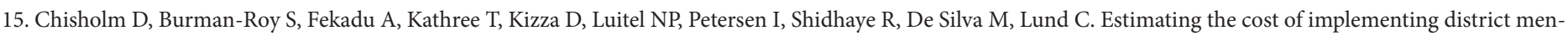
tal healthcare plans in five low-and middle-income countries: the PRIME study. The British journal of psychiatry. 2016 Jan 1;208(s56):s71-8.

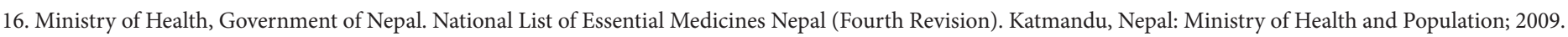
Available from, http://www.who.int/selection_medicines/country_lists/npl_eml_pdf (accessed 22 May 2017)

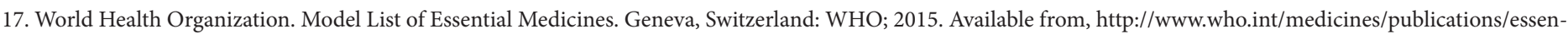
tialmedicines/EML_2015_FINAL_amended_NOV2015.pdf?ua=1 (accessed 22 May 2017)

18. Ministry of Health, Government of Nepal. Free Essential Drugs for Health Institution. In: Ministry of Health and Population GoN, editor. 2014. Available from, http://www.phinfonepal.com/2016/12/blog-post_21.html (accessed 22 May 2017) 